\title{
A nine-week-old girl with fever and seizures
}

\author{
Marie-Astrid Lefebvre MD FRCPC FAAP ${ }^{1}$, Brigitte Malette PhD², Ivan Brukner PhD ${ }^{3}$, \\ Christine Saint-Martin MD ${ }^{4}$, Jesse Papenburg MD MSc FRCPC ${ }^{1}$
}

\section{CASE PRESENTATION}

A nine-week-old girl presented to the emergency department with a $12 \mathrm{~h}$ history of fever and a $1 \mathrm{~min}$ generalized tonic-clonic seizure. Review of systems was otherwise negative. Her two-year-old sister experienced a fever and oropharyngeal ulcers two weeks before. On physical examination, she was febrile to $39.2^{\circ} \mathrm{C}$ rectal, but with otherwise normal vital signs. She was well-appearing and her examination was normal, including the neurological examination. Blood, urine and cerebrospinal fluid (CSF) specimens were obtained, and intravenous ceftriaxone, vancomycin and acyclovir were started.

Laboratory investigations showed a normal complete blood cell count and transaminase levels. CSF examination revealed 55 white blood cells/ $\mu \mathrm{L}$ ( $51 \%$ monocytes, $37 \%$ lymphocytes, $12 \%$ neutrophils), 7 red blood cells $/ \mu \mathrm{L}$, normal protein $(0.35 \mathrm{~g} / \mathrm{L})$ and normal glucose ( $2.9 \mathrm{mmol} / \mathrm{L})$. An electroencephalogram revealed active epileptiform activity over the right centroparietal regions. She was admitted to the pediatric ward and underwent magnetic resonance imaging of her head, which revealed multifocal nonenhancing lesions in the subcortical white matter of the right precentral gyrus, right cingular gyrus, right corticospinal tract, as well as the right internal capsule and thalamus (Figure 1). Bacterial cultures were without growth and antibiotics were discontinued after $48 \mathrm{~h}$. CSF herpes simplex virus (HSV) 1 and HSV 2 polymerase chain reaction (PCR) (LightCycler 2.0 HSV $1 / 2$ qualitative kit [Roche Diagnostics, Canada]) and enterovirus PCR were also negative. Repeat lumbar puncture and blood testing were performed on hospital day 3 .

\section{DIAGNOSIS}

Both the CSF and blood samples obtained on hospital day 3 returned positive results for HSV-1 using PCR, confirming a diagnosis of HSV encephalitis. Extracted DNA from the initial CSF was re-tested using a different laboratory-developed HSV 1 and HSV 2 quantitative realtime PCR assay at another reference laboratory. In retrospect, the initial CSF specimen was positive for HSV 1 (2,675 copies/mL) using this assay, as were the second CSF specimen $(21,905$ copies $/ \mathrm{mL})$ and the blood sample $(12,089$ copies $/ \mathrm{mL})$.

\section{DISCUSSION}

The present case emphasizes some of the challenges faced by clinicians in making an early diagnosis of HSV encephalitis in children. Compared with tissue culture from brain biopsy, PCR testing of the CSF has an estimated sensitivity of $98 \%$, making it the diagnostic modality of choice (1). However, several studies have demonstrated that the frequency of false-negative PCR results may be as high as $35 \%$ in children, especially during the first $72 \mathrm{~h}$ of illness $(2-5)$. This is likely explained by the lower quantity of HSV DNA in the CSF at the onset of encephalitis (2-5). Using a quantitative HSV PCR assay, we observed a 10-fold increase in HSV DNA in our patient's CSF after $72 \mathrm{~h}$ on acyclovir. This highlights how HSV CSF titres may abruptly rise in the early stages of the disease and that the first week of antiviral therapy does not significantly reduce HSV PCR sensitivity (1). Therefore, repeating a lumbar puncture if clinical suspicion persists is paramount (2-5). Moreover, CSF pleocytosis and
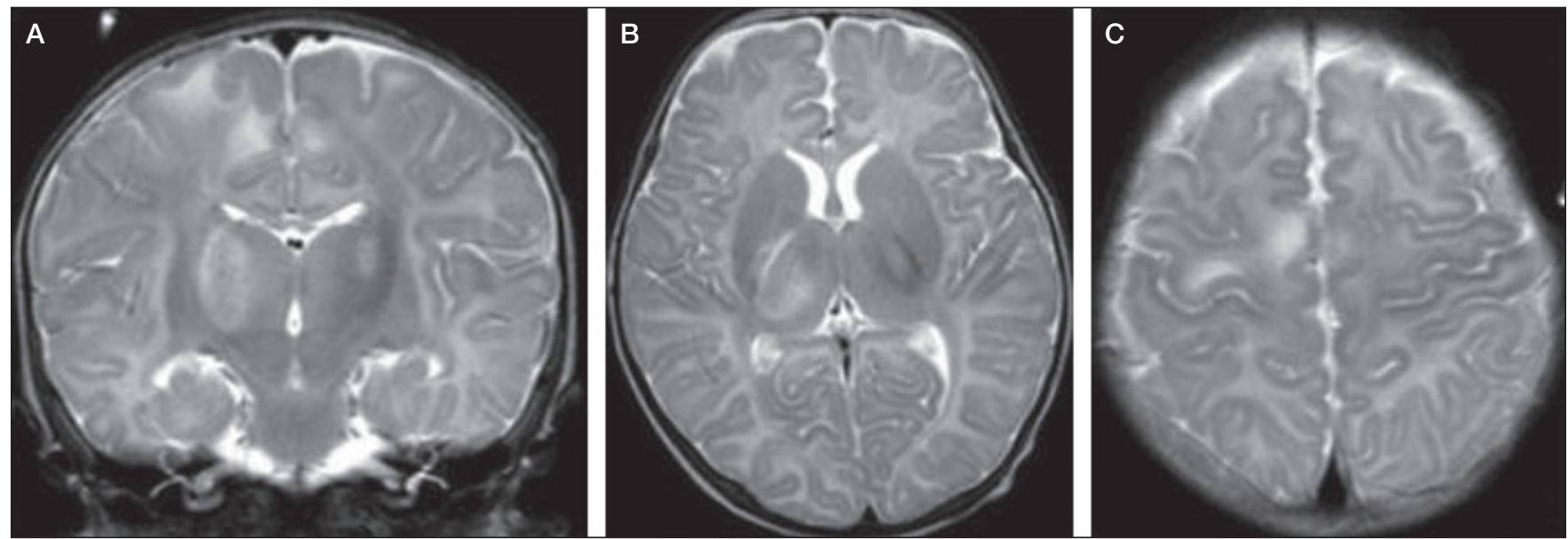

Figure 1) Coronal and axial T2-weighted images revealing increased T2 signal in the bilateral thalami (more extensive on the right [A and B]), right precentral subcortical white matter and cortex $(\mathrm{C})$

${ }^{1}$ Division of Pediatric Infectious Diseases, Departments of Pediatrics and Microbiology, Montreal Children's Hospital, McGill University Health Centre; ${ }^{2}$ Department of Microbiology, CHU Ste-Justine; ${ }^{3}$ Department of Microbiology, Jewish General Hospital; ${ }^{4}$ Department of Diagnostic Radiology, Montreal Children's Hospital, McGill University Health Centre, Montreal, Quebec

Correspondence: Dr Jesse Papenburg, Montreal Children's Hospital, 2300 Tupper Street, Room C-1246, Montreal, Quebec H3H 1P3.

Telephone 514-412-4485, fax 514-412-4494, e-mail jesse.papenburg@mail.mcill.ca 
proteinosis, although classically associated with HSV encephalitis, may be absent initially, especially in CSF samples with low levels of HSV DNA $(3,6)$. This further underscores the value of repeating CSF analyses when HSV encephalitis is suspected.

In addition to the timing of CSF sampling, other factors may influence the ability to make a microbiological diagnosis of HSV encephalitis, such as interlaboratory variability in HSV PCR sensitivities. This may be due to differences in nucleic acid extraction efficiency, laboratory proficiency or the analytical sensitivity (ie, lower limit of detection) of the assay. In the present case, despite a reported lower limit of detection of $<10$ genome equivalents per analysis (7) and no known breaches in laboratory protocol application, the first assay failed to detect HSV in our patient's initial CSF sample.

The prognostic utility of the initial level of CSF HSV DNA remains unclear. Domingues et al (8) described the use of a quantitative CSF HSV PCR in 16 adult and pediatric patients with HSV encephalitis, and found that those with $>100$ copies of HSV DNA per microlitre, before or within four days of initiation of treatment, were more likely to display lesions on a computed tomography scan of the brain and poorer neurological outcomes. Conversely, other adult studies of HSV encephalitis have not observed this association.

In cases with low/undetectable CSF HSV viral load, blood HSV PCR may serve as a useful adjunct to make an early presumptive diagnosis of HSV encephalitis, particularly in patients with a primary infection. This is possible because the pathogenesis of HSV encephalitis in neonates and young infants includes primary infection with viremia and subsequent neuroinvasion (2). In a retrospective cohort study involving 37 neonates with HSV disease, Kimura et al (9) detected HSV DNA in the blood of $73 \%$ of patients with a central nervous system infection. They also found

\section{REFERENCES}

1. Lakeman FD, Whitley RJ. Diagnosis of herpes simplex encephalitis: Application of polymerase chain reaction to cerebrospinal fluid from brain-biopsied patients and correlation with disease. National Institute of Allergy and Infectious Diseases Collaborative Antiviral Study Group. J Infect Dis 1995;171:857-63.

2. De Tiège X, Rozenberg F, Héron B. The spectrum of herpes simplex encephalitis in children. Eur J Paediatr Neurol 2008;12:72-81.

3. De Tiège X, Héron B, Lebon P, Ponsot G, Rozenberg F. Limits of early diagnosis of herpes simplex encephalitis in children: A retrospective study of 38 cases. Clin Infect Dis 2003;36:1335-9.

4. Elbers JM, Bitnun A, Richardson SE, et al. A 12-year prospective study of childhood herpes simplex encephalitis: Is there a broader spectrum of disease? Pediatrics 2007;119:e399-407.

5. Weil AA, Glaser CA, Amad Z, Forghani B. Patients with suspected herpes simplex encephalitis: Rethinking an initial negative polymerase chain reaction result. Clin Infect Dis 2002;34:1154-7. that newborns with disseminated disease had a higher serum viral load than those with central nervous system infections $\left(10^{5.9 \pm 0.6}\right.$ copies $/ \mathrm{mL}$ versus $10^{1.5 \pm 0.8}$ copies $/ \mathrm{mL}$, respectively) (9). Moreover, death and neurological sequelae among survivors were significantly associated with higher serum HSV DNA copy numbers (9). Therefore, quantitative blood HSV PCR may also show promise as a prognostic tool in neonatal HSV disease.

Nevertheless, there is little information available regarding the added value of blood HSV PCR in cases of suspected encephalitis outside of the neonatal period, in which viremia is presumed to be less common. In a case series involving 24 non-neonatal pediatric HSV infections associated with a positive blood HSV PCR, Cantey et al (10) detected HSV viremia in two immunocompetent adolescents with HSV-1 encephalitis secondary to viral reactivation, a mechanism more frequently implicated in older children (2).

Importantly, we do not recommend that a positive blood result be taken as confirmation that HSV is the causative agent of encephalitis, but rather that it be interpreted conjointly with clinical, imaging, electroencephalographic and CSF findings. Similarly, because the negative predictive value of blood HSV PCR in children outside the neonatal period is not known, a negative test should not lead to stopping empirical antiviral therapy when there is ongoing clinical suspicion for HSV encephalitis. Nevertheless, CSF HSV PCR remains the preferred diagnostic modality (1), and repeating a lumbar puncture is crucial if the initial test is negative and HSV remains a possibility.

DISCLOSURES: The authors have no financial relationships or conflicts of interest to declare. This work originated from Montreal Children's Hospital, McGill University Health Centre, Montreal, Quebec.

6. Muttalib F, Papenburg J. Absence of pleocytosis alone is insufficient to exclude encephalitis caused by herpes simplex virus in children. J Clin Microbiol 2014;52:1022.

7. Linder A, Karl G, Espy M, Smith TF, Emrich T. Rapid detection and differentiation of herpes simplex virus (HSV) types 1 and 2 in research samples with the PCR workflow system. Biochemica 2002;4:5-7.

8. Domingues RB, Lakeman FD, Mayo MS, Whitley RJ. Application of competitive PCR to cerebrospinal fluid samples from patients with herpes simplex encephalitis. J Clin Microbiol 1998;36:2229-34.

9. Kimura H, Ito Y, Futamura M, et al. Quantitation of viral load in neonatal herpes simplex virus infection and comparison between type 1 and type 2. J Med Virol 2002;67:349-53.

10. Cantey JB, Mejías A, Wallihan R, et al. Use of blood polymerase chain reaction testing for diagnosis of herpes simplex virus infection. J Pediatr 2012;161:357-61. 


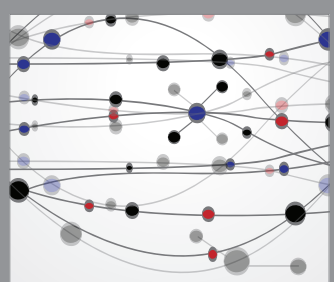

The Scientific World Journal
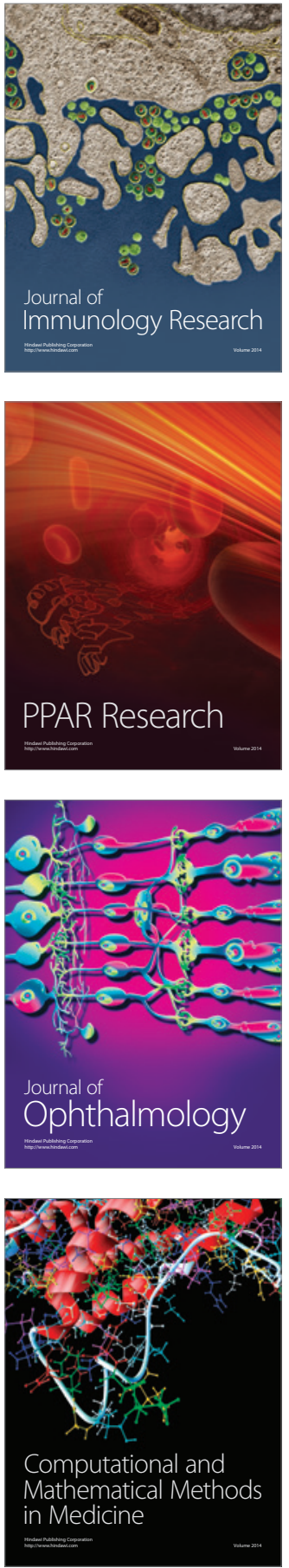

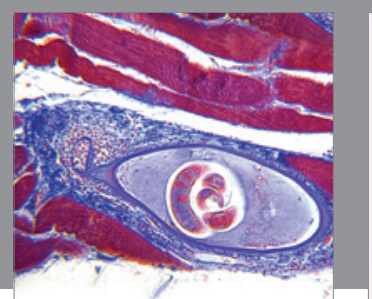

Gastroenterology Research and Practice

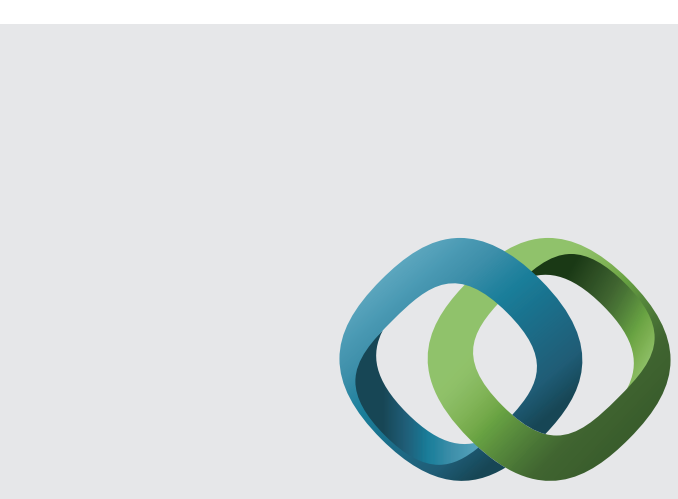

\section{Hindawi}

Submit your manuscripts at

http://www.hindawi.com
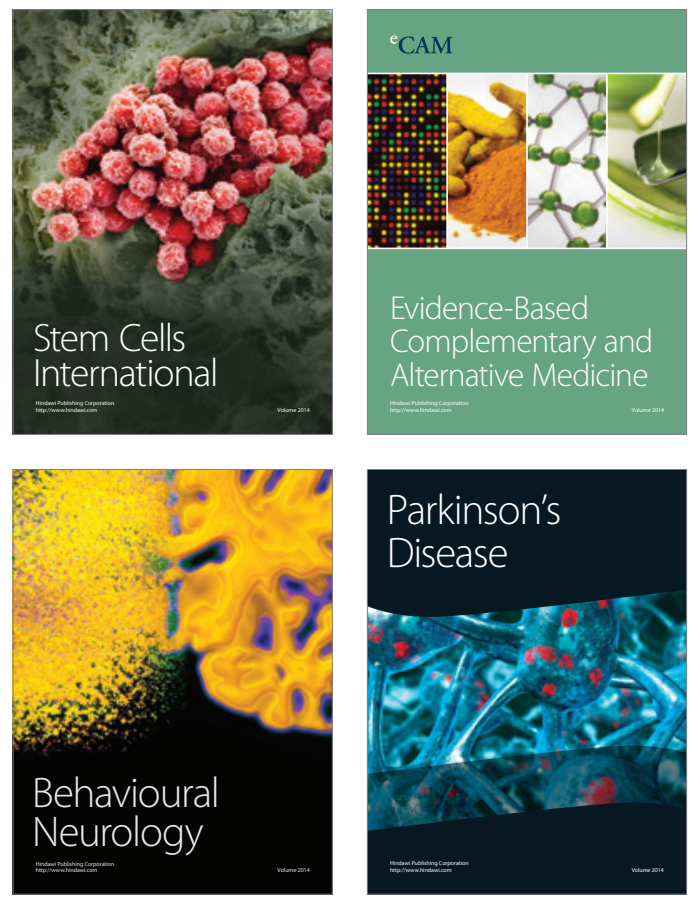
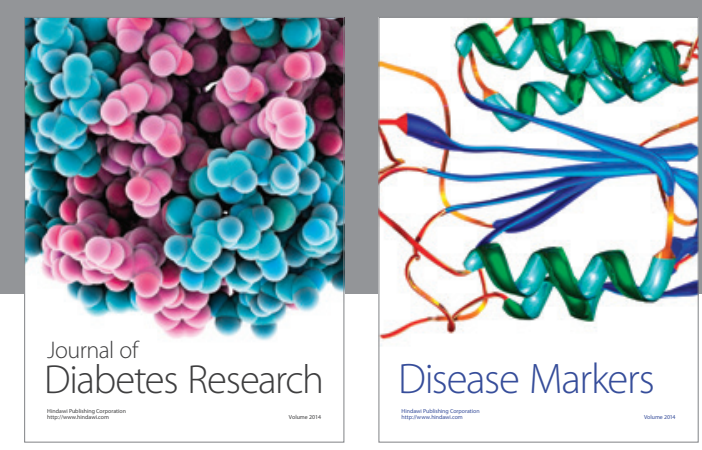

Disease Markers
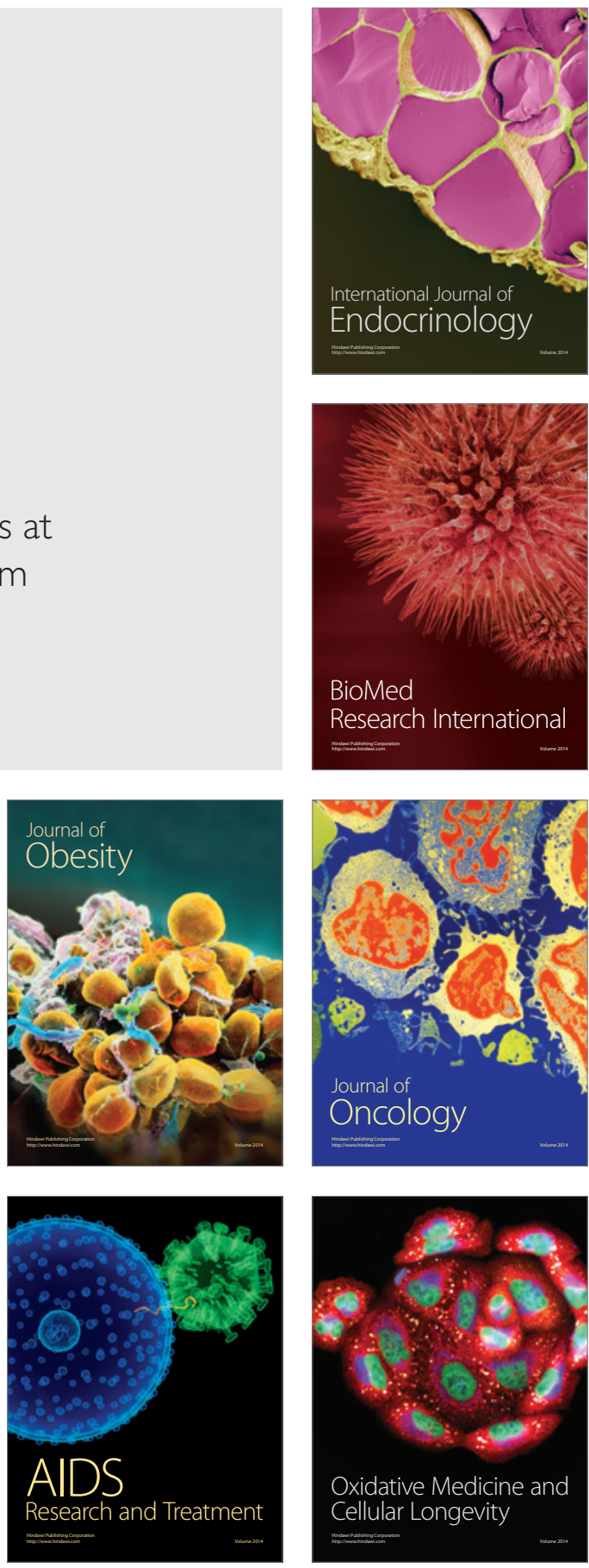